\title{
Fresnel laws at curved dielectric interfaces of microresonators
}

\author{
Martina Hentschel and Henning Schomerus \\ Max-Planck-Institut für Physik komplexer Systeme, Nöthnitzer Strasse 38, 01187 Dresden, Germany
}

(Received 23 November 2001; published 3 April 2002)

\begin{abstract}
We discuss curvature corrections to Fresnel's laws for the reflection and transmission of light at a nonplanar refractive-index boundary. The reflection coefficients are obtained from the resonances of a dielectric disk within a sequential-reflection model. The Goos-Hänchen effect for curved light fronts at a planar interface can be adapted to provide a qualitative and quantitative extension of the ray model which explains the observed deviations from Fresnel's laws.
\end{abstract}

DOI: $10.1103 /$ PhysRevE.65.045603

PACS number(s): 42.25.Gy, 03.65.Sq, 42.15.-i, 42.60.Da

The fabrication of lasing microresonators $[1,2]$ and optomechanical microdevices [3] has generated a surge of interest in the confinement and propagation of light in small dielectric structures. Some understanding has been achieved from the ray optics of these systems, complemented by Fresnel's laws of refraction and reflection at the interfaces, e.g., in order to identify and describe the relevant resonator modes $[1,2]$. Fresnel's laws give the probability of reflection and refraction of plane electromagnetic waves at planar interfaces of media with different refractive index $n$. Microresonators, however, often are so small that the curvature of their boundary cannot be neglected.

In this paper we investigate, in the limit of large wave numbers, the corrections to the Fresnel coefficients that appear due to the curvature of the dielectric interface. The reflection coefficients are obtained via a sequential-reflection model [4] from the resonance widths of a microresonator, which are analytically accessible for large wave numbers. The deviations from Fresnel's laws are most noticeable around the critical angle for total internal reflection, $\chi_{c}$ $=\arcsin (1 / n)$ (where the refractive index of the surrounding medium is set to unity) and amount to a systematic reduction of the reflection probability.

The reduction of the reflection probability is conventionally related to tunneling escape at the curved interface. In view of the previous success of the ray model, which often is desired to be retained for its simplicity, we provide an alternative qualitative and quantitative explanation of the deviations by incorporating into this model the Goos-Hänchen effect [5-10]. This effect results from the interference of rays in a beam with slight variations of the angle of incidence and consists of a shift of the effective plane of reflection. At a curved interface, it turns out that the reflection probability is then reduced because the angle of incidence at the effective interface is smaller than at the physical interface.

There is evidence obtained in the context of quantummechanical scattering problems [11] that incorporating the Goos-Hänchen effect is equivalent to a semiclassical approximation. Also the effect has been used in Ref. [12] to explain the decreased spacing of resonances observed in experiments with dielectric spheres, in terms of an effective optical size of the cavity that is larger than its physical size. Our work can be seen as complementary to this previous study, because we are concerned with the resonance widths, not only the resonance energies.
Curvature corrections to Fresnel coefficients have been addressed in the past in a number of works, by applying various techniques; see, for example, Refs. [13-16]. The works closest in spirit to the present paper are those that employ the complex ray method, e.g., to describe light rays approaching a disk from outside $[15,16]$.

Although we restrict our discussion to circular interfaces, the results for the reflection coefficients should be applicable to microresonators of general shape as long as locally the curvature can be approximated as a constant.

According to Fresnel's laws, a plane electromagnetic wave incident on a planar dielectric interface with angle of incidence $\chi$ is reflected with the polarization-dependent coefficients [17]

$$
\begin{aligned}
& R^{\mathrm{TM}}=\frac{\sin ^{2}(\chi-\eta)}{\sin ^{2}(\chi+\eta)}=\left|\frac{n \cos \chi+\cos \eta}{n \cos \chi-\cos \eta}\right|^{2}, \\
& R^{\mathrm{TE}}=\frac{\tan ^{2}(\chi-\eta)}{\tan ^{2}(\chi+\eta)}=\left|\frac{\cos \chi+n \cos \eta}{\cos \chi-n \cos \eta}\right|^{2},
\end{aligned}
$$

where TM (TE) signifies transverse polarization of the magnetic (electric) field at the interface and $\eta=\arcsin (n \sin \chi)$ is the direction of the refracted beam (according to Snell's law).

Let us compare the Fresnel coefficients with the reflection coefficients at a curved interface with radius of curvature $r_{c}$. Their angular dependence can be conveniently obtained from the energies and widths of resonance states in a twodimensional circular disk of radius $r_{c}$. In this geometry the two possible polarization directions decouple and angular momentum (quantum number $m$ ) is conserved. We introduce polar coordinates $r$ and $\phi$ and denote the (complex) wave number by $k$. We will concentrate on the case close to geometric optics $\operatorname{Re} k r_{c} \gg 1$.

The resonance states are obtained by matching the wave field proportional to $J_{m}(n k r) e^{i m \phi}$ inside the disk (with the Bessel function $J$ ) at $r=r_{c}$ to the wave field proportional to $H_{m}^{(1)}(k r) e^{i m \phi}$ outside the disk (with the Hankel function $H^{(1)}$ ), where the matching conditions follow from Maxwell's equations:

$$
J_{m}\left(n k r_{c}\right) H_{m}^{(1)^{\prime}}\left(k r_{c}\right)=n J_{m}^{\prime}\left(n k r_{c}\right) H_{m}^{(1)}\left(k r_{c}\right)
$$

for TM polarization, and 


$$
n J_{m}\left(n k r_{c}\right) H_{m}^{(1) \prime}\left(k r_{c}\right)=J_{m}^{\prime}\left(n k r_{c}\right) H_{m}^{(1)}\left(k r_{c}\right)
$$

in the TE case (primes denote derivatives). Given a complex solution $k$, the angle of incidence is obtained from the real part by comparing the angular momentum in the ray picture $\left(L=n p r_{c} \sin \chi, p=\hbar \operatorname{Re} k\right)$ and in the wave picture ( $L$ $=\hbar m)$,

$$
\sin \chi=\frac{m}{n \operatorname{Re} k r_{c}},
$$

while the reflection probability

$$
R=\exp \left(4 n \operatorname{Im} k r_{c} \cos \chi\right)
$$

follows from the imaginary part of $k$ because it determines the intensity decay rate $\gamma_{I}=-2 \operatorname{Im} k c$ (with $c$ the velocity of light outside the disk), which in turn can be related to $R$ by a ray-based model of sequential reflections [4]: After $s$ reflections the wave intensity inside the disk drops to a fraction $R^{s}$ of the initial intensity. Reflections occur with a rate $\gamma_{s}$ $=c /\left(2 n r_{c} \cos \chi\right)$, where the denominator is the optical path length between consecutive reflections. After time $t$, we relate $R^{t \gamma_{s}}=e^{-t \gamma_{I}}$, directly yielding Eq. (5).

Because the discrete set of resonance energies obtained from Eqs. (2) and (3) is meaningful only for the disk, let us first derive analytical expressions for the resonance width, as a function of a continuous resonance energy, that smoothly interpolate between these solutions. It is interesting to note [4] that one cannot simply expand Eqs. (2) and (3) in $\operatorname{Im} k$ when $k$ is not close to an exact solution. Moreover, for TE polarization $\operatorname{Im} k$ will diverge at the Brewster angle if it is calculated by inserting the Fresnel coefficient (1b) into Eq. (5). In order to achieve a more accurate expansion we separate out the problematic term and cast Eqs. (2) and (3) into the form

$$
\frac{J_{m}^{\prime}\left(n k r_{c}\right)}{J_{m}\left(n k r_{c}\right)}=\mathcal{F}\left(k r_{c}\right)
$$

with

$$
\mathcal{F}^{\mathrm{TE}}(x)=n \frac{H_{m}^{(1)^{\prime}}(x)}{H_{m}^{(1)}(x)}, \quad \mathcal{F}^{\mathrm{TM}}(x)=n^{-2} \mathcal{F}^{\mathrm{TE}}(x),
$$

depending on the polarization. In both cases, $\mathcal{F}\left(k r_{c}\right)$ is a slowly varying complex function of its argument, and the argument can be taken real because $\operatorname{Re} k \gg|\operatorname{Im} k|$. The logarithmic derivative of Bessel functions, however, is a rapidly fluctuating function, and its dependence on $\operatorname{Im} k$ has to be worked out carefully. This can be achieved by approximation by tangents $[18]$ :

$$
\begin{gathered}
\frac{J_{m}^{\prime}\left(n k r_{c}\right)}{J_{m}\left(n k r_{c}\right)}=-\tan \alpha \cos \chi, \\
\alpha=m \cot \chi+m \chi-m \frac{\pi}{2}-\frac{\pi}{4}+i n \operatorname{Im} k r_{c} \cos \chi,
\end{gathered}
$$

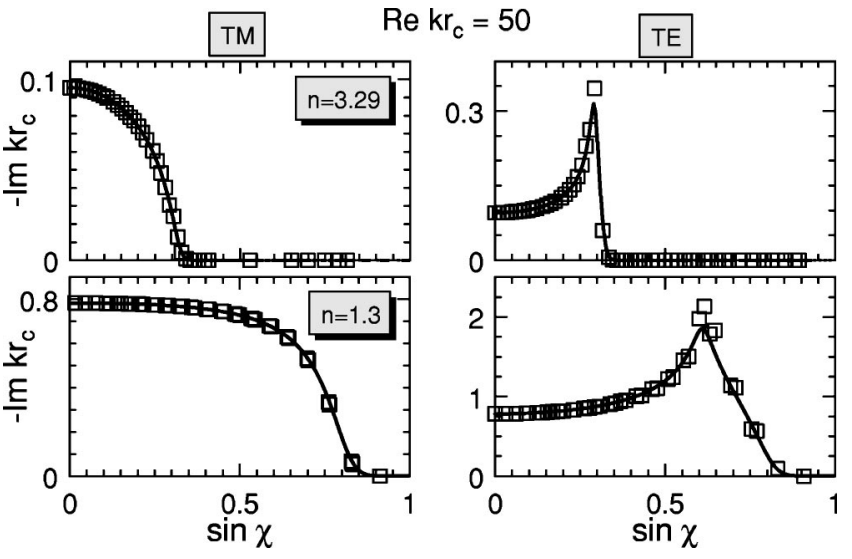

FIG. 1. Resonance widths $-\operatorname{Im} k r_{c}$ for a dielectric disk with Re $k r_{c}=50$ and a refractive index $n=3.29$ (upper panel) and $n$ $=1.3$ (lower panel), for TM and TE polarized light, as a function of the angular momentum [parametrized according to Eq. (4)]. The analytical result from Eqs. (10),(11) (solid curves) is compared with the exact results from Eqs. (2),(3) (squares).

where $\chi$ is given as a function of $\operatorname{Re} k$ by Eq. (4). We expanded $\alpha$ linearly in $\operatorname{Im} k$ and neglected terms of order $\left(\operatorname{Re} k r_{c}\right)^{-1}$. Equation (6) can now be solved for $\alpha$, without any further approximation. From the imaginary part one deduces

$$
-\operatorname{Im} k r_{c}=\frac{1}{n \cos \chi} \operatorname{Im} \arctan \frac{\mathcal{F}}{\cos \chi} .
$$

Although this can already be taken as the final result, we may further insert the uniform approximation [18]

$$
\mathcal{F}^{\mathrm{TE}}=i n \cos \eta\left[1+\frac{1}{\sin ^{2} \eta}\left(\frac{K_{2 / 3}(z)}{K_{1 / 3}(z)}-1\right)\right],
$$

and similarly for $\mathcal{F}^{\mathrm{TM}}=\mathcal{F}^{\mathrm{TE}} / n^{2}$, with the modified Bessel function $K$, the angle of refraction $\eta$ (which is a complex number for $\left.\chi>\chi_{c}\right)$, and $z=-i \operatorname{Re} k r_{c} \cos ^{3} \eta /\left(3 \sin ^{2} \eta\right)$. In Fig. 1 we illustrate that Eqs. (10) and (11) agree very well with the exact solutions of Eqs. (2) and (3), even close to the Brewster angle for TE polarization, and interpolate smoothly in between.

The angular dependence of the reflection coefficients can now be obtained by combining Eqs. (5) and (10), giving the final result

$$
R=\left|\frac{\cos \chi+i \mathcal{F}}{\cos \chi-i \mathcal{F}}\right|^{2}
$$

Figures 2 and 3 show the results for the two values $\operatorname{Re} k r_{c}$ $=50$ and $\operatorname{Re} k r_{c}=150$, respectively. Deviations from Fresnel's laws [Eq. (1)] are most visible around the critical angle $\chi_{c}=\arcsin (1 / n)$ where the reflection coefficients increase rapidly as the regime of total internal reflection is approached. The correction consists not only in a broadening of the transition interval, but most notably also in a shift of this transition region toward higher angles of incidence, resulting in a systematic reduction of the reflection coefficient. 


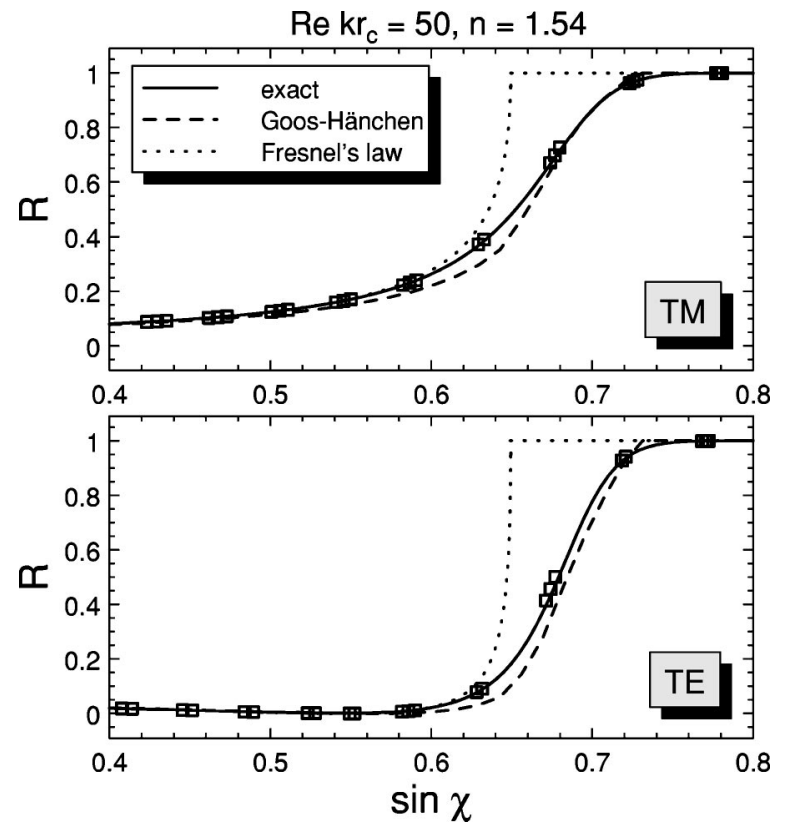

FIG. 2. Reflection coefficients $R$ in a dielectric disk with $\operatorname{Re} k r_{c}=50$. The solid curve is the analytical result Eq. (12), which smoothly interpolates between the exact solutions of Eqs. (2) and (3) with real part close to 50 [squares, translated into angulardependent reflection coefficients by Eqs. (4) and (5)]. The dashed curve is the result of incorporating the Goos-Hänchen effect into a ray model (assuming for TE polarization that the shift is the same as for TM polarization, for reasons explained in the text). The dotted curve is Fresnel's law [Eq. (1)].
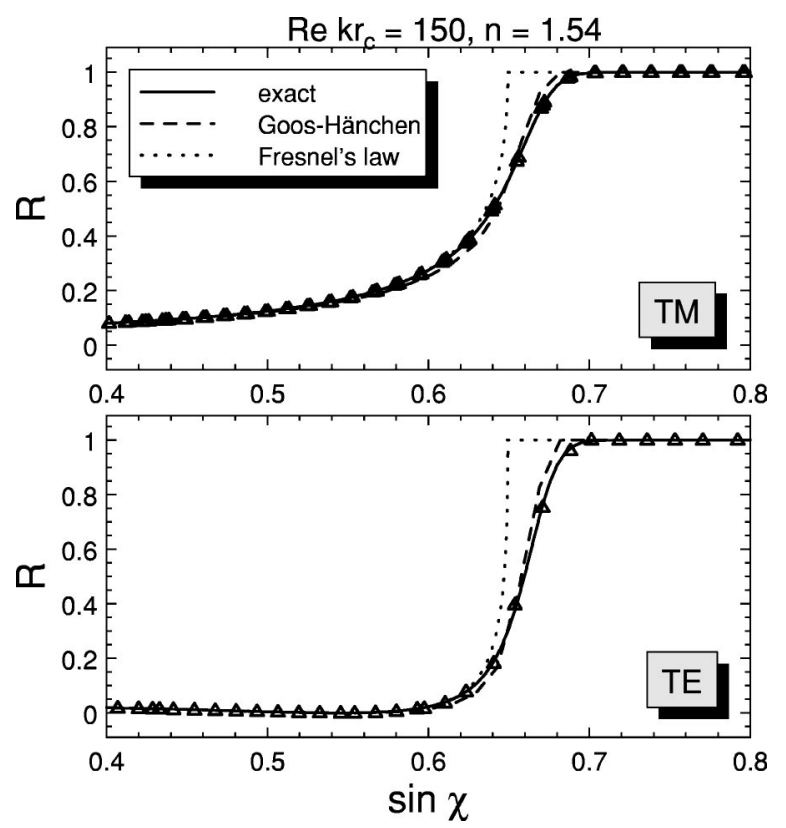

FIG. 3. Same as Fig. 2 but for a wave number $\operatorname{Re} k r_{c}=150$. The result based on the Goos-Hänchen effect is almost indistinguishable from the exact result.

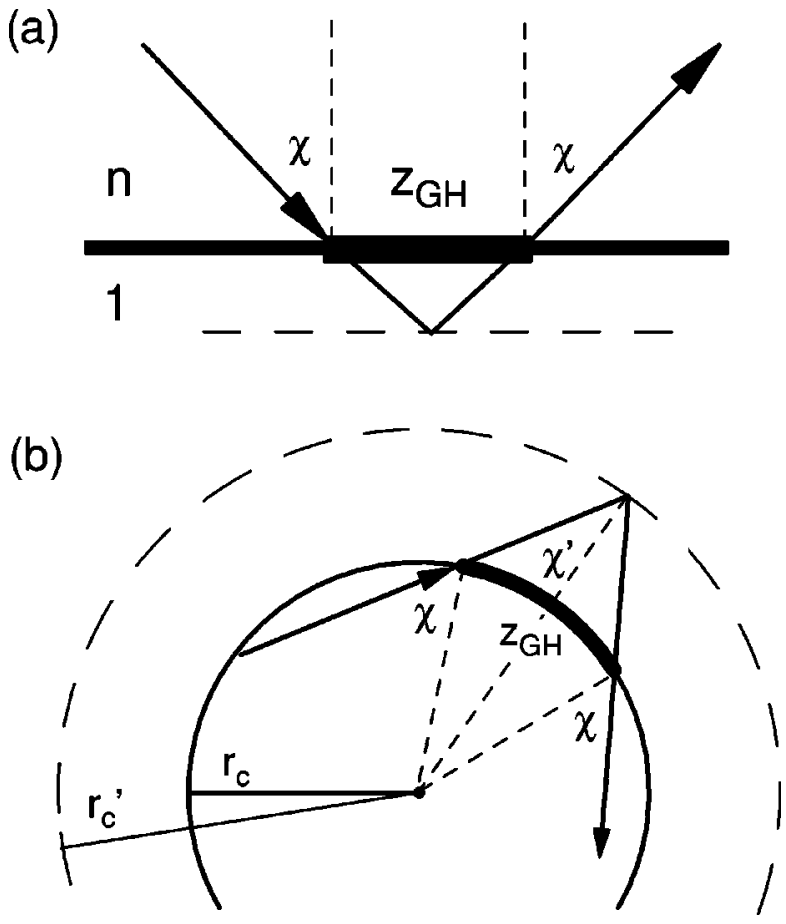

FIG. 4. (a) Goos-Hänchen shift at a planar interface. An incident beam containing contributions from plane waves of slightly different angles of incidence $\chi$ appears to be reflected at a position that is shifted a distance $z_{\mathrm{GH}}$ away from the point of incidence. Alternatively, one can think of the beam as being reflected at a shifted interface indicated by the dashed lines. (b) Goos-Hänchen effect at a curved interface. The reflection seems to occur at an interface of larger curvature radius $r_{c}^{\prime}>r_{c}$ under a smaller angle $\chi^{\prime}<\chi$ of incidence.

The deviations from Fresnel's laws in Figs. 2 and 3 increase as $\operatorname{Re} k r_{c}$ is reduced, that is, the more noticeable the curvature of the interface is. On the other hand, in the zerowavelength limit $\operatorname{Re} k r_{c} \rightarrow \infty$ of geometric optics any interface appears planar, and Fresnel's laws should apply without modification. Indeed, it can be seen that they are recovered from Eq. (12) when the approximation by tangents is also applied to the Hankel functions in $\mathcal{F}$, resulting in $\mathcal{F}^{\mathrm{TM}}$ $=i n^{-1} \cos \eta, \mathcal{F}^{\mathrm{TE}}=i n \cos \eta$. The deviations close to the critical angle are directly related to the breakdown of this approximation when the argument of the Hankel functions becomes smaller than the index. As we will discuss now, the curvature corrections to Fresnel's coefficients can be obtained within a minimal extension of the ray picture when the Goos-Hänchen effect is taken into account (the result obtained is given by the dashed curves in Figs. 2 and 3).

The Goos-Hänchen effect [5-10] refers to the displacement of the reflected beam when the incident beam consists of rays with slight variations of the angle of incidence, and arises because each ray experiences a slightly different phase shift when it is reflected. As is illustrated in Fig. 4(a), the lateral shift along the interface can also be interpreted as resulting from a displacement of the effective plane of reflection (a signature of this displacement is the increased Wigner delay time which has recently been measured at metallic gratings [19]). 
Since at a planar interface the reflection law is not affected by parallel displacement and is fulfilled for the mean angles of incidence, the reflection coefficients are not affected by the Goos-Hänchen effect-the only consequence of the slight variation of angles is that their angular dependence is smeared out. However, the situation changes at a curved interface [11] as shown in Fig. 4(b). The intersection of the incident and the laterally shifted reflected rays defines an effective boundary of radius $r_{c}^{\prime}>r_{c}$. We now can assume that the ray is specularly reflected at the effective boundary, resulting in a smaller effective angle of incidence $\chi^{\prime}<\chi$, and evaluate the Fresnel reflection coefficients (1) at this smaller angle $\chi^{\prime}$. This angular shift has been observed in Ref. [20] for rays approaching the disk from outside. Since the reflection probability is then reduced this qualitatively explains the observed deviations from Fresnel's laws in Figs. 2 and 3.

For a quantitative comparison we need the distribution of angles of incidence $P(\chi)$, which can be related to the radial width proportional to $r_{c}\left(n \operatorname{Re} k r_{c}\right)^{-2 / 3}$ of the caustic in the radial wave field $J_{m}(n k r)$. (The same distribution of angles will also be used to smooth out the reflection coefficients as is appropriate even for the planar interface.) The variation of angles of incidence arises from the intrinsic curvature of the beam wave front and also directly from the curvature of the interface-note that both mechanisms should contribute equally to the Goos-Hänchen effect. We found that for our purposes $P(\chi)$ is sufficiently well approximated by that of a Gaussian beam of half-width $\sigma=r_{c}\left(n \operatorname{Re} k r_{c}\right)^{-2 / 3} \ll r_{c}$. It is good to observe that this Gaussian beam geometry does not put us into conflict with the finite disk size while at the same time leaving us in the paraxial regime $n \operatorname{Re} k \sigma$ $=\left(n \operatorname{Re} k r_{c}\right)^{1 / 3} \gg 1$. For TM polarization the effective radius of curvature $r_{c}^{\prime}$ can then be calculated by applying the formulas of Ref. [7] (which are lengthy expressions and hence not given here). The result of this Goos-Hänchen-effectbased approach is presented as the dashed curves in the upper panels of Figs. 2 and 3. There is good agreement with the exact results obtained within the sequential-reflection model. For TE polarization and the chosen refractive index, the analytic result in the literature becomes applicable only for $\operatorname{Re} k r_{c} \gtrsim 1000$. Surprisingly (see however Ref. [21]), in the current situation nice agreement is found by simply assuming that the Goos-Hänchen shift is identical to that is the TM case, as is indicated by the dashed curves in the lower panels of Figs. 2 and 3. (For Re $k r_{c} \gtrsim 1000$, however, it is appropriate to work with the correct TE formulas.)

In conclusion, we investigated the reflection coefficients at a curved refractive-index boundary by relating them to resonances in a circular dielectric disk and derived analytic expressions valid in the limit of large wave numbers. The deviations from Fresnel's laws can be explained within geometric optics by incorporating the Goos-Hänchen effect. In this work we concentrated on the wave field confined by reflection inside the microcavity. The Goos-Hänchen shift also affects the wave field outside the cavity $[11,16,20]$, because the modified angle of incidence results in a change of the angle of refraction. It is desirable to investigate the implications on the remarkable emission directionality of noncircular devices $[1,2]$, which sometimes departs substantially from what is expected from geometric optics [22].

We gratefully acknowledge helpful discussions with M. Berry, S. W. Kim, J. U. Nöckel, K. Richter, R. Schubert, and J. Wiersig.
[1] J.U. Nöckel and A.D. Stone, Nature (London) 385, 45 (1997).

[2] C. Gmachl, F. Capasso, E.E. Narimanov, J.U. Nöckel, A.D. Stone, J. Faist, D.L. Sivco, and A.Y. Cho, Science 280, 1556 (1998)

[3] A. Ashkin, Optical Trapping and Manipulation of Neutral Particles Using Lasers (World Scientific, Singapore, 2001).

[4] M. Hentschel and J.U. Nöckel, in Quantum Optics of Small Structures, edited by D. Lenstra, T.D. Visser, and K.A.H. van Leeuwen (Edita KNAW, Amsterdam, 2000).

[5] F. Goos and H. Hänchen, Ann. Phys. (Leipzig) 1, 333 (1947).

[6] K. Artmann, Ann. Phys. (Leipzig) 8, 270 (1951).

[7] H.M. Lai, F.C. Cheng, and W.K. Tang, J. Opt. Soc. Am. A 3, 550 (1986).

[8] A.W. Snyder and J.D. Love, Optical Waveguide Theory (Chapman and Hall, London, 1983).

[9] B.R. Horowitz and T. Tamir, J. Opt. Soc. Am. 61, 586 (1971).

[10] H.K.V. Lotsch, Optik (Stuttgart) 32, 116 (1970); 32, 189 (1970); 32, 299 (1971); 32, 553 (1971).

[11] J. Herb, P. Meerwald, M.J. Moritz, and H. Friedrich, Phys. Rev. A 60, 853 (1999).

[12] D.Q. Chowdhury, D.H. Leach, and R.K. Chang, J. Opt. Soc.
Am. A 11, 1110 (1994).

[13] A.W. Snyder and J.D. Love, IEEE Trans. Microwave Theory Tech. MTT-23, 134 (1975).

[14] A.L. Cullen, Ann. Telecommun. 31, 359 (1976).

[15] Y.Z. Ruan and L.B. Felsen, J. Opt. Soc. Am. A 3, 550 (1986).

[16] N. Fiedler-Ferrari, H.M. Nussenzveig, and W.J. Wiscombe, Phys. Rev. A 43, 1005 (1991).

[17] M. Born, and E. Wolf, Principles of Optics (Pergamon Press, Oxford, 1999).

[18] I.S. Gradshteyn and I.M. Ryzhik, in Table of Integrals, Series, and Products, edited by A. Jeffrey (Academic Press, San Diego, 1994).

[19] D. Chauvat, O. Emile, F. Bretenaker, and A. Le Floch, Phys. Rev. Lett. 84, 71 (2000).

[20] N.H. Tran, L. Dutriaux, Ph. Balcou, A. Le Floch, and F. Bretenaker, Opt. Lett. 20, 1233 (1995).

[21] A. Haibel, G. Nimtz, and A.A. Stahlhofen, Phys. Rev. E 63, 047601 (2001).

[22] N. B. Rex, H. E. Tureci, H. G. L. Schwefel, R. K. Chang, and A. D. Stone, Phys. Rev. Lett. 88, 09410 (2002). 